\title{
RESOURCE REVIEWS
}

MedEdPORTAL. Association of American Medical Colleges, $655 \mathrm{~K}$ Street, Northwest, Suite 100, Washington, DC 20001-2399; mededportal@aamc.org; https:// www.mededportal.org; free website.

\section{PURPOSE}

According to the Association of American Medical Colleges (AAMC), the mission of MedEdPORTAL is to "[promote] educational scholarship and collaboration by facilitating the open exchange of peer-reviewed health education teaching and assessment resources" [1].

\section{DESCRIPTION}

Celebrating its ten-year anniversary in 2015, MedEdPORTAL is the premier resource for locating and publishing free, open educational resources in the health sciences. Similar to MERLOT <http:// merlot.org $>$, MedEdPORTAL is an online repository of educational materials such as, but not limited to, lectures, online modules, clinical cases, and teambased learning exercises as well as assessments including quizzes, tests, and survey instruments. While both provide free access to peer-reviewed educational materials, MERLOT and MedEdPORTAL differ significantly in scope. Whereas MERLOT caters to all disciplines and targets all education levels from kindergarten to continuing education, MedEdPORTAL is solely dedicated to health sciences education. MedEdPORTAL focuses on medical education but covers many health sciences disciplines, including dentistry, nursing, and pharmacology. Materials for all levels of health sciences education (undergraduate, graduate, and continuing education for professionals) are also found in MedEdPORTAL.

Though the product targets educators in the health sciences, anyone can register for a free AAMC account to access resources and submit items for publication in MedEdPORTAL. However, some material, such as student assessments with accompanying answers, may be protected and require verification from your institution. MedEdPortal will ask you to provide the name and email address of your institution's human resources personnel; they will contact them to verify whether you are a student, faculty member, or staff. This process ensures that students do not have ready access to sensitive course materials.

MedEdPORTAL is divided into three main components: MedEdPORTAL Publications, iCollaborative, and the CE Directory. Whether you are looking for materials to adopt at your own institution or planning to publish, each component fulfills both needs splendidly.

\section{MedEdPORTAL Publications}

MedEdPORTAL Publications is the peer-reviewed section, with over 2,000 materials as of January 2016. MedEdPORTAL Publications accepts all forms of educational resources, which undergo a rigorous screening and peer-review process to be accepted, just like traditional journal articles. Publications is an excellent source for teaching and assessment materials for you to use at your own institution. Some of these materials are appropriate for librarians, including resources on information literacy and evidence-based medicine training in the health sciences. Why reinvent the wheel when it has been created, peer reviewed, and made openly accessible for your use?

\section{iCollaborative}

iCollaborative houses MedEdPORTAL's non-peer-reviewed resources, serving as a temporary place to share in-progress projects and gather feedback from the professional community. In addition to the education resources that you can find in Publications, iCollaborative also includes conference posters, oral presentations, and other gray literature that may not be accessible elsewhere. Materials in iCollaborative are stored for three years before being removed by MedEdPORTAL; authors can request that material be removed sooner. It is assumed that many of these resources will eventually be submitted to Publications. Currently, nearly 3,000 materials have been uploaded into iCollaborative.

\section{CE Directory}

The CE Directory is simply that: a directory currently linking to over 500 online continuing education (CE) activities for professionals in the health sciences. All activities listed in the CE Directory can be completed entirely online, and most are in the field of medicine, with accompanying credits for 
the American Medical Association Physician's Recognition Award (PRA). Though there is no fee to access the MedEdPORTAL CE Directory, the host offering the activity may charge fees. The CE Directory is a great resource to recommend to all educators in the health sciences, both to encourage lifelong learning and to fulfill $\mathrm{CE}$ requirements in their disciplines.

In addition to these three components, MedEdPORTAL is also currently developing several themed subcollections of resources, including Interprofessional Education (IPE), Undergraduate Science (uScience), and a Directory and Repository of Educational Assessment Measures (DREAM). For more information, visit $<$ https://www.mededportal.org/ about/initiatives/>.

\section{SEARCHING MEDEDPORTAL}

In Publications, iCollaborative, or the CE Directory, MedEdPORTAL has a single search box in the upper right-hand corner of each web page, where you can search using a few keywords or leave the search box blank to retrieve all resources associated with the section you are searching. You cannot, however, search across all three sections at once. You can filter searches by specialty, topic, instructional or assessment methods, intended audience, format, and more, but no other advanced search features are available. You can also adjust the number of search results per page, how the results are sorted (most recent or most relevant), and show or hide abstracts. Overall, however, search capabilities are limited in MedEdPORTAL, and simply browsing the results list may prove more effective.
On an individual resource's web page, you will find detailed information about the resource, including its abstract, author information, link to download the material, and copyright information. As MedEdPORTAL is an open repository, all materials are offered under a Creative Commons license selected by the authors; terms are clearly indicated for each resource. When downloading materials, you will be required to login to your AAMC account, asked your status and purpose for downloading the material (for publication tracking purposes), and presented with a compressed file to download. Each compressed file will include either an instructor's guide (in publications prior to November $15,2015)$ or an educational summary report (ESR), the actual educational materials (usually in the form of PowerPoint files, Word documents, links to websites, or video files), and a copyright summary page listing how you may use the material.

\section{PUBLISHING IN MEDEDPOR- TAL}

You may submit resources to all three sections of MedEdPORTAL. Submissions to iCollaborative and the CE Directory are fairly straightforward. iCollaborative has no peer-review process, but the submitted material must be related to health sciences education to be eligible. Activities included in the CE Directory must be able to be completed entirely online and be accredited by a health professional CE provider.

Submissions to Publications undergo an extensive review process. All submissions must include: an ESR (basically a mini-manuscript) and all files needed to implement the educational activity, as well as proof that the submission meets Glassick's six criteria for scholarship [2], usually described in the ESR. Each submission is screened by MedEdPORTAL staff before peer review to ensure that it meets minimal scholarship criteria and does not contain any copyrighted material. As all publications are freely available online, all images, videos, and so on must be either in the public domain or under a Creative Commons license, or the authors must get copyright permission from the original author. The screening process can often prove more frustrating than the peer-review process but may provide an excellent opportunity for librarians to partner with authors at their institutions to help them identify alternative resources to include in each submission. The average time from passing through screening to a final decision is approximately three months.

One major drawback to MedEdPORTAL is that it is currently not indexed in any database; however, the AAMC is working to get it indexed in PubMed. MedEdPORTAL does provide usage data to authors about their publications, accessible at any time within the author's AAMC account. The usage report tracks how many times the publication has been downloaded and for what purpose it was downloaded (teaching, self-learning, curriculum development, and assessment) as well as the country of origin, institution, and status (faculty, researcher, librarian, student, and so on) of those who downloaded the report. These data can be used as evidence of research impact for promotion and tenure purposes. For example, MedEdPORTAL publications are encouraged and even formally included in the promotion and tenure guidelines of all faculty, including librarians, at the reviewer's med- 
ical school and valued as scholarly output.

For health sciences librarians, MedEdPORTAL offers three major opportunities: (1) to locate and adapt existing materials for our own instruction and assessment purposes; (2) to use our role as educators as a form of scholarship by publishing in MedEdPORTAL in addition to traditional journal publications; and (3) to increase collaborative relations with our faculty and clinical colleagues through instruction and guidance on successfully navigating the MedEdPORTAL submission process. I look forward to seeing our profession embrace MedEdPORTAL as a valuable source of educational material and a viable publishing venue.

Stephanie M. Swanberg, MSI, AHIP, swanberg@oakland.edu, Medical Library, Oakland University William Beaumont School of Medicine, Rochester, MI

\section{REFERENCES}

1. Association of American Medical Colleges. MedEdPORTAL: mission and vision [Internet]. The Association; 2016 [cited 25 Jan 2016 ]. <https://www. meded portal.org/about/ missionandvision/>.

2. Glassick CE, Huber MT, Maeroff GI. Scholarship assessed: evaluation of the professoriate. San Francisco, CA: Jossey-Bass; 1997.

DOI: http://dx.doi.org/10.3163/15365050.104.3.020
Google Scholar. Google, 1600 Amphitheatre Parkway, Mountain View, CA 94043; cost: free; https://scholar.google.com.

Scopus. Elsevier, 1600 John F. Kennedy Boulevard, Suite 1800, Philadelphia, PA 19103-2398; contact publisher for quote; https:// www.elsevier.com/solutions/ scopus.

\section{GOOGLE SCHOLAR AND SCOPUS FOR FINDING GRAY LITERATURE PUBLICATIONS}

Some authors have suggested that it is challenging to give a conclusive definition of what constitutes gray literature, but gray literature is defined as "Information produced on all levels of government, academia, business and industry in electronic and print formats not controlled by commercial publishing, i.e., where publishing is not the primary activity of the producing body" [1]. Main publishers of gray literature include research institutions, universities, international, national and local authorities, and industrial firms. Types of gray literature include dissertations, conference proceedings and papers, and technical or research reports [2].

Gray literature, and conference papers in particular, are an essential source for emerging approaches to new medical interventions. Often, conference papers might be the only source of information for new drugs and medical interventions and the only source of preliminary findings from clinical trials. The Cochrane Handbook of Searching notes that reviewers should include information from unpublished studies, and the failure to identify trials noted in conference proceedings and other sources of gray literature might affect the results of a systematic review [3]. As a result, conference papers provide an essential contribution to the evidence base, especially for systematic reviews; however, it can be challenging to locate gray literature sources, including conference papers and guidelines. Most databases such as MEDLINE do not index gray literature publications, guidelines, or best practices that are not published in the journal literature. Also, databases might only index conference papers when the complete proceedings have been published as part of a journal. Even when the proceedings are published in an issue of a journal, the indexing for individual abstracts of papers may be severely limited. Both Google Scholar and Scopus, however, index a variety of types of gray literature. This review discusses the search functionality and search options in both tools for finding gray literature publications, with a focus on conference papers.

\section{Google Scholar}

Google Scholar is a freely available search engine that searches across a wide range of academic sources. Recent estimates of Google Scholar's content have found that it indexes over 160 million items, including journal articles, book chapters, dissertations, and conference papers [4]; however, Google Scholar's website does not provide any information regarding the number of conference papers included in the academic search engine [5]. Google Scholar is often cited as a source for gray literature, especially in the search methodology for systematic reviews and in library research guides on gray literature. 